\title{
Diagnosis Error: Carcinoma or Primary Breast Lymphoma? A Case Report and Literature Review*
}

\author{
Hinde El Fatemi ${ }^{1 \#}$, Ihsane Souaf ${ }^{1}$, Soufia El Jayi ${ }^{2}$, Kaoutar Moumna ${ }^{1}$, My Abdelilah Melhouf², \\ Nadia Sqali ${ }^{3}$, Afaf Amarti ${ }^{1}$ \\ ${ }^{1}$ Department of Pathology, Hassan II Teaching Hospital, Fez, Morocco \\ ${ }^{2}$ Department of Gynecology, Hassan II Teaching Hospital, Fez, Morocco \\ ${ }^{3}$ Department of Radiology, Hassan II Teaching Hospital, Fez, Morocco \\ Email: "hinde0012@hotmail.com
}

Received September 25, 2012; accepted October 28, 2012; accepted November 8, 2012

\begin{abstract}
Introduction: Breast involvement by non-Hodgkin lymphoma is particularly rare in women. We describe the case of a patient with a rapidly growing, nodule in the right breast. On ultrasonography, the nodule was suspicious for breast carcinoma. Case presentation: A breast biopsy from a 73-year-old Moroccan women answered invasive ductal carcinoma grade 3 from Elston and Ellis. Patey was performed. Microscopic examination showed lymphoid proliferation confirmed on immunohistochemical analysis. Our patient was treated with chemotherapy. Conclusions: The rarity of breast lymphomas, and the problems related to the diagnosis and therapeutic choices with these tumors require molecular techniques in association with classical histological diagnosis.
\end{abstract}

Keywords: Breast Cancer; Breast Primary Lymphoma; Immunohistochemistrey; Diagnosis

\section{Introduction}

The term "primary breast lymphoma" (PBL) is used to define a malignant lymphoma primarily occurring in the breast in the absence of previously detected lymphoma localizations [1]. PBL is a rare disease, accounting for only $0.4 \%-0.5 \%$ of all breast malignancies, $0.38 \%$ $0.7 \%$ of all non-Hodgkin lymphomas (NHL), and $1.7 \%$ $2.2 \%$ of extranodal NHL [2,3]. Despite the clinical and radiographic similarities between breast lymphoma and carcinoma, the prognosis, as reported in the literature, varies, as do the applied treatment modalities, which include surgery, radiotherapy and chemotherapy used alone or in combination. In this report we describe a rare case of breast PBL whose diagnosis was primary carcinoma. The authors discussed the problems related to the diagnosis and therapeutic choices with these tumors require molecular techniques in association with classical histological diagnosis.

\section{Case Presentation}

A 70-year-old Moroccan woman presented with a right breast mass present for 4 months. She discovered a painless mass on her right breast from self-breast examination. She had not a history of familial breast cancer.

\footnotetext{
*Non-financial competing interests.

\#Corresponding author.
}

Physical examination revealed a fixed, firm, painless 8 $\mathrm{cm}$ mass at the upper inner quadrant of the right breast. There was warmth, erythema, ecchymosis, skin retraction overlying the mass, nipple retraction, and discharge. There was a movable, rubbery, painless $1 \mathrm{~cm}$ right axillar lymph node, but no other adenopathy was found. The lung sounds were clear. She had no signs of upper airway obstruction. Oxygen saturation was $100 \%$ on ambient air. Liver and spleen were not palpable. The remainder of the examination was unremarkable. Mammography and ultrasonography showed a breast mass ACR 5 (Americain College of Radiology). A Fine Needle Aspiration (FNA) revealed poorly differentiated carcinoma without immunohistochemical confirmation. Patey was performed. Macroscopic examination showed well circumscribed tumour measuring $8 \mathrm{~cm}$ in diameter. On cut surface, the neoplastic tissue is white-grey, firm with focal hemorrhagic foci. Pathology examination showed diffuse malignant proliferation mimicking lobular carcinoma (Figure 1). Tumours cells were negative for cytokeratin and E-cadherine, positive for CD20 (Figure 2), MUM1, and bcl-2 and they were negative for CD3. Thoracic, abdominal and pelvic CT scans did not reveal tumor masses or nodes. She was diagnosed with primary breast diffuse large B cell lymphoma stage II (node positive). She responded well to chemotherapy. At months of follow-up, there is no evidence of disease recurrence. 


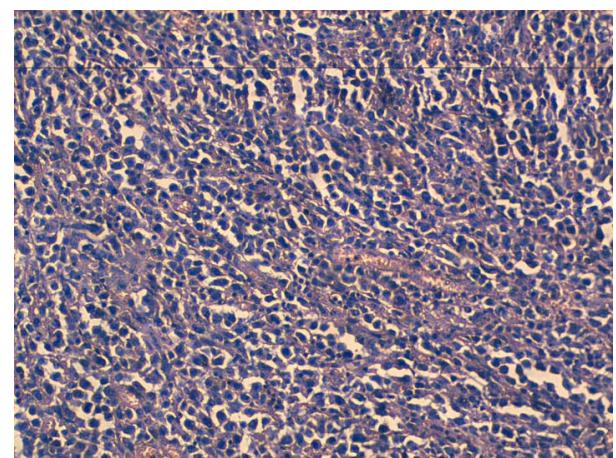

Figure 1. HES X 20: Diffuse malignant proliferation mimicking lobular carcinoma.

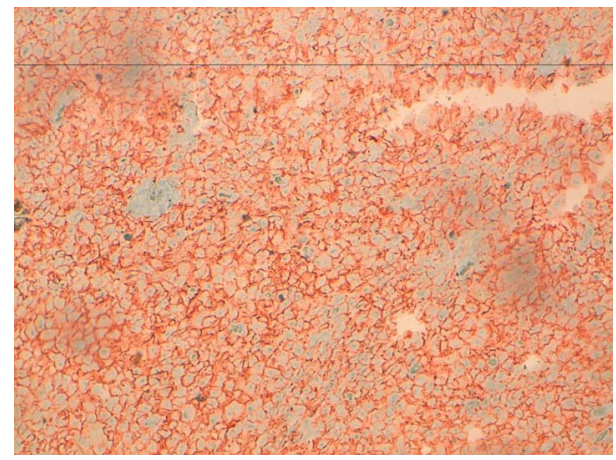

Figure 2. Tumours cells positivity for CD20.

\section{Discussion}

The median age of patients with diagnosed PBL ranges from 60 to 65 years [1-4]. The disease occurs almost exclusively in women. Bilateral breast involvement accounts for $11 \%$ of all breast lymphomas [5] or $5 \%$ according to Ryan et al. [6]. This rare situation is especially observed during pregnancy or postpartum, suggesting that tumour growth is influenced by hormonal stimulation. Breast lymphoid cells probably originate in mucosa-associated lymphoid tissue (MALT) [7]. PBL may also originate from lymphatic tissue present within the breast adjacent to ducts and lobules, or from intramammary lymph nodes $[8,9]$. More than $80 \%$ of PBL are B-cell lymphomas (DLBCL), mostly CD20+. The most frequent histopathologic types are: diffuse large B-cell lymphoma (DLBCL) which accounts for up to $50 \%$ of all PBL, follicular lymphoma (FL) - 15\%, MALT lymphoma-12.2\%, Burkitt's lymphoma (BL) and Burkittlike lymphoma-10.3\% [10]. Other histological types of PBL include marginal zone lymphoma (MZL), small lymphocyticlymphoma (SLL), and anaplastic large cell lymphoma (ALCL). A painless mass is the most common presenting sign in PBL occurring in approximately 61\% of cases, especially (48\%) in the external superior quadrant. Other symptoms and signs are the following: local pain-12\%, local inflammation-11\%, palpable lymph nodes - $25 \%$, incidental mammography finding-12\%
[1]. Mammographic findings are non specific. Most of the lesions are oval-shaped (71\%) and high-density (90.9\%) masses. The masses revealed by ultrasound examination are single (75\%), circumscribed $(50 \%)$, microlobulated (37.5\%) and oval (50\%). The echo pattern of the mass is usually hypoechoic (87\%). No masses have spiculated margins or calcifications [11]. Fine needle aspiration, core biopsy and excisional biopsy are effective techniques used in the evaluation of breast nodules and axillary lymph nodes. However, histological, immunohistochemical and, sometimes, genetic studies are necessary for establishing the diagnosis. Wiseman and Liao [12] reported three criteria for diagnosis of primary NHL of the breast. Adequate pathological evaluation, presence of both mammary tissue and lymphoma infiltrate in close association, and exclusion of either systemic lymphoma or previous extramammary lymphoma. In gross pattern, the lesion appears as a well circumscribed tumour of varying size, up to $20 \mathrm{~cm}$ in largest diameter. On cut surface, the neoplastic tissue is white to white-grey, soft or firm, with occasional haemorrhagic or necrotic foci [13]. In histological examination, diffuse large B-cell lymphoma is characterized by a diffuse pattern of infiltration of breast tissue by large lymphoma cells varying in appearance from quite uniform to pleomrphic. The lymphoma cells resemble centroblasts or immunoblasts. The nuclei are oval, indented or even lobated, usually with distinct, single or multiple nucleoli, and the amount of cytoplasm is variable. Mitoses are usually numerous, various numbers of cells are apoptotic and nectrotic foci may be found. Lymphoma cells are often admixed with smaller reactive lymphocytes of B or $\mathrm{T}$ type; Macrophages may be prominent, imparting a "starry sky" appearance to the tumour. In some cases pseudofollicullar structures are seen due to selective infiltration of ductal-lobular units. Adjacent mammary tissue may exhibit lobular atrophy or lymphocytic lobulitis; the latter may be prominent and widespread, featuring lymphocytic mastopathy. Lymphoma cells are immunoreactive for CD20, CD79a and melanoma associated antigen (mutated) 1(MUM1) protein, and negative for CD5, CD3; the proliferative index evaluated by Ki67 immunostaining was higher than 50\%. [13] Mastectomy has been a common component of PBL therapy for decades and remains a frequent treatment choice in some reports. Several studies found that mastectomy offered no benefit in the treatment of primary breast lymphoma $[5,14]$. Ideally, surgery should be limited to a biopsy to establish the correct histological diagnosis, leaving the treatment with curative intent to radiotherapy and chemotherapy. In our case, mastectomy was performed. Jennings et al. [10] reviewed all published PBL reports from 3 decades (1972-2005). Patient demographics, such as survival, recurrence and time to follow-up, were recorded, in addition to surgical, radiation and/or chemotherapeutic treat- 
ment(s). A total of 465 patients were found. The age range was 17 to 95 years (mean, 54 years). Follow-up ranged from 1 to 288 months (mean, 48 months). DLBCL was the most common histological diagnosis (53\%). Treatment by mastectomy offered no survival benefit. Treatment that included radiation therapy in stage I patients (node negative) showed benefit in both survival and recurrence rates. Treatment that included chemotherapy in stage II patients (node positive) showed benefit in both survival and recurrence rates. Diseasefree survival was $44.5 \%$ overall. In conclusion, mastectomy offers no benefit to the treatment of PBL. Nodal status predicts outcome and guides the optimal use of radiation and chemotherapy. Treatment of primary breast lymphomas follows treatment recommendations for lymphomas of the same stage and histology in other locations. The choice of chemotherapeutic regimen should be based upon histological subtype, disease extent and the individual patient. There are many controversies about prognostic factors for patients with PBL. One of these is the histological subtype of PBL. According to Ryan et al. [6], a favourable International Prognostic Index (IPI) score, the use of anthracycline-containing chemotherapy, and radiotherapy (RT) are significantly associated with longer overall survival (OS). Similarly, Jeanneret-Sozzi [1] confirmed, by means of univariate analysis, that favourable prognostic factors are: early stage (IE), conservative surgery, RT administration and combined modality treatment. Multivariate analyses also confirm that the early stage and use of RT are favourable prognostic factors. Jennings et al. [10] in a Cox regression model, analyzing the tumour size and node status, revealed that the node status is the best single predictor of survival. The most common chemotherapy agents used in PBL have been those in the CHOP regimen. Strategies to minimize the cardiac toxicity risks associated with doxorubicin-containing combination regimens, by using adjuvant radiation therapy, have been reported for non-Hodgkin's lymphoma of sites other than breast and may be important [15]. Rituximab has been Registered for treatment of DLBCL and follicular lymphoma FL. To our knowledge, there are no valuable data that confirm improvement after the addition of rituximab in patients with DLBCL- and FL-PBL. The efficacy of rituximab is well documented in other CD20+ B cell lymphomas. Therefore, prospective trials should be pursued to check the impact of using rituximab on clinical outcome and patterns of relapse. However, it will be difficult due to low incidence of PBL in the general population.

\section{Conclusion}

In conclusion, most authors recommend that aggressive forms of PBL should have central nervous system (CNS) prophylaxis even in the early stages. In our opinion, in some forms of PBL combination chemotherapy may improve the outcome and significantly reduce the future risk of CNS relapse.

\section{REFERENCES}

[1] W. Jeanneret-Sozzi, A. Taghian, R. Epelbaum, et al., "Primary Breast Lymphoma: Patient Profile, Outcome and Prognostic Factors. A Multicentre Rare Cancer Network Study,” BMC Cancer, Vol. 8, 2008, p. 86. doi:10.1186/1471-2407-8-86

[2] C. S. Ha, P. Dubey, L. K. Goyal, M. Hess, F. Cabanillas and J. D. Cox, "Localized Primary Non-Hodgkin Lymphoma of the Breast," American Journal of Clinical Oncology, Vol. 21, No. 4, 1998, pp. 376-380. doi:10.1097/00000421-199808000-00012

[3] D. Meerkotter, G. Rubin, F. Joske, P. Angunawela and A. Khalafallah, "Primary Breast Lymphoma: A Rare Entity," Journal of Radiology Case Reports, Vol. 5, No. 5, 2011, pp. 1-9.

[4] M. Topalovski, D. Crisan and J. C. Mattson, "Lymphoma of the Breast. A Clinicopathologic Study of Primary and Secondary Cases," Archives of Pathology \& Laboratory Medicine, Vol. 123, No. 12, 1999, pp. 1208-1218.

[5] K. Ganjoo, R. Advani, M. R. Mariappan, A. McMillan and S. Horning, "Non-Hodgkin Lymphoma of the Breast," Cancer, Vol. 110, No. 1, 2007, pp. 25-30. doi:10.1002/cncr.22753

[6] G. Ryan, G. Martinelli, M. Kuper-Hommel, et al., "Primary Diffuse Large B-Cell Lymphoma of the Breast: Prognostic Factors and Outcomes of a Study by the International Extranodal Lymphoma Study Group,” Annals of Oncology, Vol. 19, No. 2, 2008, pp. 233-241. doi:10.1093/annonc/mdm471

[7] S. H. Kim, M. P. Ezekiel and R. Y. Kim, "Primary Lymphoma of the Breast: Breast Mass as an Initial Symptom," American Journal of Clinical Oncology, Vol. 22, No. 4, 1999, pp. 381-383. doi:10.1097/00000421-199908000-00011

[8] A. H. Dao, R. B. Adkins Jr. and A. D. Glick, "Malignant Lymphoma of the Breast: A Review of 13 Cases," The American Journal of Surgery, Vol. 58, No. 12, 1992, pp. 792-796.

[9] J. R. Zack, S. G. Trevisan and M. Gupta, "Primary Breast Lymphoma Originating in a Benign Intramammary Lymph Node,” American Journal of Roentgenology, Vol. 177, No. 1, 2001, pp. 177-178.

[10] W. C. Jennings, R. S. Baker, S. S. Murray, et al., "Primary Breast Lymphoma: The Role of Mastectomy and the Importance of Lymph Node Status," Annals of Surgery, Vol. 245, No. 5, 2007, pp. 784-789. doi:10.1097/01.sla.0000254418.90192.59

[11] C. Y. Lyou, S. K. Yang, D. H. Choe, B. H. Lee and K. H. Kim, "Mammographic and Sonographic Findings of Primary Breast Lymphoma,” Clinical Imaging, Vol. 31, No. 4, 2007, pp. 234-238. doi:10.1016/j.clinimag.2007.02.028

[12] R. Franco, A. Fernández-Vázquez, M. Mollejo, M. A. 
Cruz, F. I. Camacho, J. F. García, M. Navarrete and M. A. Piris, "Cutaneous Presentation of Follicular Lymphomas,” Modern Pathology, Vol. 14, No. 9, 2001, pp. 913919. doi:10.1038/modpathol.3880411

[13] A. Fattaneh, Tavassoli, P. Deville, et al., "Tumours of the Breast and Female Genital Organs. Pathology and Genitics," International Agency for Research on Cancer, Lyon, 2004.

[14] M. H. Lbrahim and S. Singletary, "Surgical Management of Primary Lymphoma of the Breast,” Annals of Surgery, Vol. 214, No. 6, 1991, pp. 724-726.

[15] T. P. Miller, S. Dahlberg, J. R. Cassady, et al., "Chemotherapy alone Compared with Chemotherapy plus Radiotherapy for Localized Intermediate- and High-Grade NonHodgkin's Lymphoma," The New England Journal of Medicine, Vol. 339, 1998, pp. 21-26. doi:10.1056/NEJM199807023390104 